\title{
Atypical Posterior Reversible Encephalopathy Syndrome in Puerperium
}

\author{
N. Morellia, $\quad$ S. Gori ${ }^{b}$ M.C. Michelassi ${ }^{c}$ M. Falorni ${ }^{b} \quad$ G. Cafforio ${ }^{b} \quad$ M.C. Bianchic \\ M. Cosottini ${ }^{b, c} \quad$ G. Orlandi ${ }^{b} \quad$ L. Murri $^{b} \quad$ A. Tartaglione ${ }^{a}$

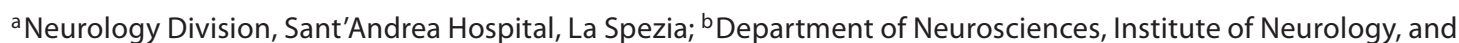 \\ 'Unit of Neuroradiology, Pisa University, Pisa, Italy
}

Dear Sir,

Posterior reversible encephalopathy syndrome (PRES) is a clinical and radiological entity characterized by headache, visual loss and epileptic seizures in a severe arterial hypertension setting due to hypertension-inducing diseases, such as renal diseases or eclampsia [1,2]. Moreover, PRES can also be caused by hypertension-inducing treatments (i.e. erythropoietin, blood transfusion or immunosuppressants) [1].

Brain MRI typically documents vasogenic edema involving the occipitoparietal regions [3] as a result of loss of cerebral autoregulation related to hypertensive endothelial dysfunction and disruption of blood-brain barrier with increased permeability [4]. If PRES is diagnosed at the right time, both clinical signs and neuroradiological pattern are frequently reversible, whereas delayed diagnosis and treatment can lead to ischemic or hemorrhagic lesions with permanent neurological damage [4].

Edema can rarely involve the brainstem or the hemispheric deep structures. In this case, the resulting syndrome is called atypical PRES [5]. Misdiagnosis is a frequent event and involves vertebrobasilar ischemia, cerebral venous thrombosis and metabolic diseases. Here, we report two unusual cases of atypical PRES during puerperium with good outcome.

\section{Case Report 1}

A 37-year-old woman with no significant past medical history presented on the 4 th day of puerperium with sudden headache and severe bilateral visual disturbance (blurring and teichopsia) followed by isolated generalized tonic-clonic seizure. No other impairment was observed at neurological examination. Arterial blood pressure was consistently elevated $(180 / 110 \mathrm{~mm} \mathrm{Hg})$. Blood and urine routine assays were normal, and no proteinuria was detected during both the pregnancy and puerperium. Cranial CT scan was unremarkable, whereas MRI FLAIR and FSE$\mathrm{T}_{2}$ sequences revealed scattered areas of increased signal in subcortical occipital and posterior parietal white matter, and more confluent areas of signal hyperintensity in the grey matter of the striatum, in the external and internal capsule bilaterally and in the upper brainstem (fig. 1a, b). Diffusion-weighted images (DWI) revealed signal isointensity or slight hyperintensity of the affected areas with an increase in the apparent diffusion coefficient (ADC) indicating vasogenic edema. MR venography was also performed to evaluate the deep venous system, and resulted normal.

The patient was treated with labetalol $20 \mathrm{mg}$ i.v. for $2 \mathrm{~min}$ followed by $40 \mathrm{mg}$ i.v. at 10 -min intervals until normalization of arterial blood pressure values. Moreover, antiepileptic treatment with clonazepam was performed. Headache disappeared during the following days and no other neurological deficit occurred. Brain MRI performed 7 days later documented normalization of the pathological findings (fig. 1c, d).

\section{Case Report 2}

A 27-year-old woman with no significant past medical history presented with headache, mental confusion and generalized tonic-clonic seizures on the 7th day of puerperium. No other impairment was observed at neurological examination. She had severe arterial hypertension (240/115 $\mathrm{mm} \mathrm{Hg}$ ). Blood and urine routine assays were normal, and no proteinuria was detected during both the pregnancy and puerperium. A CT scan of the brain showed subarachnoid hemorrhage in the upper right frontal spaces (fig. 2a). Cranial MRI documented basal ganglia and subcortical matter hyperintensity on FLAIR and FSE$\mathrm{T}_{2}$ sequences (fig. $2 \mathrm{~b}$ ). On DWI, these areas had increased signal $\left(\mathrm{T}_{2}\right.$ shine-through phenomenon); fig. 2c) with increased ADC value (fig. $2 \mathrm{~d}$ ). MR angiography performed to exclude signs of postpartum angiopathy and MR venography to evaluate deep venous system were both normal. The pa-

\section{KARGER \\ Fax +41 613061234 \\ E-Mail karger@karger.ch}

www.karger.com (c) 2008 S. Karger AG, Basel 0014-3022/08/0594-0195\$24.50/0

Accessible online at: www.karger.com/ene
Nicola Morelli, MD

Sant'Andrea Hospital, Neurology Division

Via Vittorio Veneto, 197

IT-19124 La Spezia (Italy)

Tel. +39 0187553 111, Fax +39 0187533 433, E-Mail n.morelli@inwind.it 

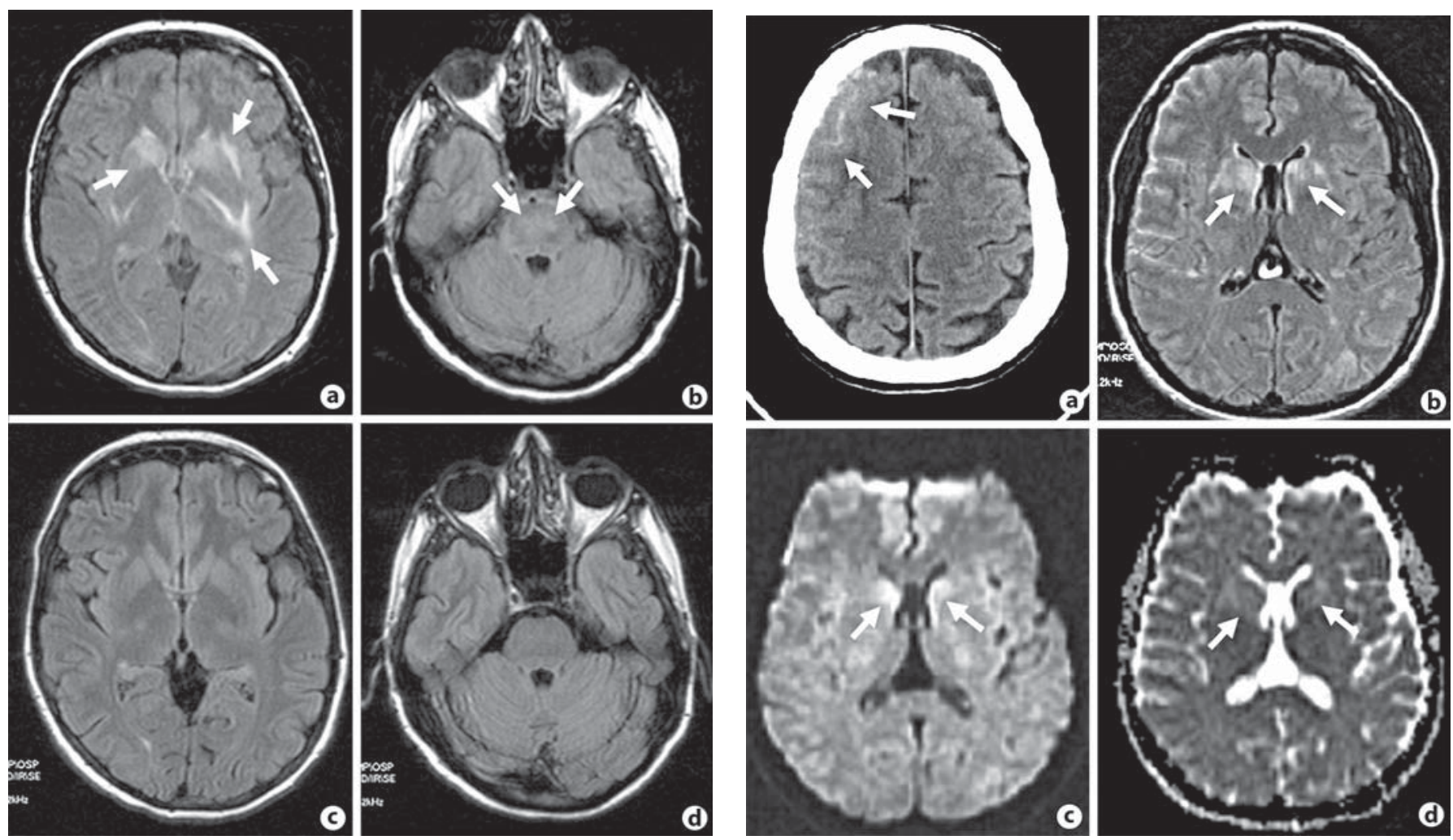

Fig. 1. FLAIR and FSE- $T_{2}$ sequences show increased signal in the subcortical occipital and the posterior parietal white matter more extensively in the grey matter of the striatum, in the external and internal capsule bilaterally (a) and in the upper brainstem (b). c, d Brain MRI control performed 7 days later documents normalization of the pathological findings.

Fig. 2. a A noncontrast cranial CT scan reveals subarachnoid hemorrhage in the upper right frontal spaces. MRI shows basal ganglia and subcortical matter hyperintensity on FLAIR sequence (b) and increased signal (c) with increased ADC value on DWI sequences indicating vasogenic edema $(\mathbf{d})$.

tient was treated with clonazepam, labetalol i.v. until normalization of arterial blood pressure. Nimodipine ( $1.5 \mathrm{mg} / \mathrm{h}$ i.v.) and magnesium sulphate ( $3 \mathrm{~g}$ i.v. followed by continuous infusion at $1 \mathrm{~g} / \mathrm{h}$ ) was administered and both the clinical and the neuroradiological picture became normal after few days.

\section{Discussion}

We have reported 2 young women with atypical PRES occurring in puerperium, to date described only in few isolated cases $[5,6]$. Our MRI findings were characterized by a vasogenic edema involving both deep cerebral structures (the basal nucleus and internal capsules) and the brain stem (pons), data suggestive of atypical PRES in late postpartum eclampsia
Moreover, we observed a favorable outcome despite the fact that one of our cases presented concomitant subarachnoid hemorrhage. Servillo et al. [7] reported a 27year-old woman in the 38 th week of gestation that developed PRES with persistent hypertension complicated by fatal subarachnoid hemorrhage and died 2 weeks later. In our case, the patient with subarachnoid hemorrhage presented normalization of arterial pressure values and full resolution of both clinical and radiological signs.

Neuroimaging has confirmed the diagnosis of PRES, demonstrating reversible hyperintensity spreading out from posterior brain regions. The occurrence of DWI and $\mathrm{ADC}$ map changes in posterior reversible encephalopathy is well documented [8], and can successfully differentiate PRES from early cerebral ischemia, thus playing a pivotal role in treatment deci- sions. Indeed, as soon as the diagnosis of PRES has been reached, early treatment with antihypertensive and antiepileptic drugs should be started. If a delay in diagnosis and treatment of PRES occurs, permanent neurological damage or even death can result from cerebral infarction or hemorrhage [3]. A reversible cerebral herniation in the setting of eclampsia associated with PRES [9], and death by cerebral herniation in a woman with cerebral edema complicating eclampsia [10] have been described. Favorable outcome has been reported in 2 patients with prevalent brainstem involvement [11] and following lacunar lesions and in a case of hypertensive brainstem encephalopathy with massive edema of the pons [12].

Puerperium represents a risk factor for PRES probably because arterial hypertension and high vulnerability of cerebral au- 
toregulation lead to the development of cerebral vasogenic edema.

Misdiagnosis frequently occurs with syndromes sharing similar MRI findings such as hypoglycemic encephalopathy, extrapontine myelinolysis and deep venous thrombosis. Hyperintensity on DWI may also occur in ischemic stroke, even though it is not specific for it [13] because the hyperintensity on DWI may be associated also with nonischemic lesion. Therefore, the DWI sequences should always be com- bined with the study of the ADC map that can be made through the logarithmic analysis of the MRI images. The ADC map allows us to differentiate the vasogenic edema (increased signal in ADC, i.e. PRES) from the cytotoxic edema (decreased signal in ADC, i.e. ischemic lesion).

Therefore, we stress the use of the ADC map to identify typical and atypical PRES, differentiating those disorders from others affecting the brain by cytotoxic edema (i.e. ischemic stroke).

\section{References}

1 Striano P, Striano S, Tortora F, De Robertis E, Palumbo D, Elefante A, Servillo G: Clinical spectrum and critical care management of posterior reversible encephalopathy syndrome (PRES). Med Sci Monit 2005;11:549_ 553.

2 Schaefer PW, Buonanno FS, Gonzalez RG, Schwam LH: Diffusion-weighted imaging discriminates between cytotoxic and vasogenic oedema in a patient with eclampsia. Stroke 1997;28:1082-1085.

3 Hinchey J, Chaves C, Appignani B, Breen J, Pao L, Wang A, Pessin MS, Lamy C, Mas JL, Caplan LR: A reversible posterior leukoencephalopathy syndrome. N Engl J Med 1996; 334:494-500.

4 Vaughan CJ, Delanty N: Hypertensive emergencies. Lancet 2000;356:411-417.
Moreover, when headache or visual disorders occur in puerperium, brain MRI examination is recommended for early diagnosis and treatment of PRES.

\section{Acknowledgement}

The authors are grateful to Dr. Michelangelo Mancuso for his critical reading of the manuscript.
5 Ahn KJ, You WJ, Jeong SL, Lee JW, Kim BS, Lee JH, Yang DW, Son YM, Hahn ST: Atypical manifestations of reversible posterior leukoencephalopathy syndrome: findings on diffusion imaging and ADC mapping. Neuroradiology 2004;46:978-983.

6 Manfredi M, Beltramello A, Bongiovanni LG, Polo A, Pistoia L, Rizzuto N: Eclamptic encephalopathy: imaging and pathogenetic considerations. Acta Neurol Scand 1997;96: 277-282.

7 Servillo G, Striano P, Striano S, Tortora F, Boccella P, De Robertis E, Rossano F, Briganti F, Tufano R: Posterior reversible encephalopathy syndrome (PRES) in critically ill obstetric patients. Intensive Care Med 2003; 29:2323-2326

8 Ay H, Buonanno FS, Schafer PW, Le DA, Wang B, Gonzalez RG, Koroshetz WJ: Posterior leukoencephalopathy without severe hypertension: utility of diffusion-weighted MRI. Neurology 1998;51:1369-1376.
9 Belogolovkin V, Levine SR, Fields MC, Stone JL: Postpartum eclampsia complicated by reversible cerebral herniation. Obstet Gynecol 2006;107:442-445.

10 Cunningham FG, Twickler D: Cerebral oedema complicating eclampsia. Am J Obstet Gynecol 2000;182:94-100.

11 Morello F, Marino A, Cigolini M, Cappellari F: Hypertensive brain stem encephalopathy: clinically silent massive oedema of the pons. Neurol Sci 2001;22:317-320.

12 Kitaguchi H, Tomimoto H, Miki Y, Yamamoto A, Terada K, Satoi H, Kanda M, Fukuyama $\mathrm{H}$ : A brainstem variant of reversible posterior leukoencephalopathy syndrome. Neuroradiology 2005;47:652-656.

13 Lovblad KO, Laubach HJ, Baird AE, Curtin F, Schlaug G, Edelman RR, Warach S: Clinical experience with diffusion-weighted MR in patients with acute stroke. AJNR Am Neuroradiol 1998;19:1061-1066. 